\title{
Metal Abundances and Ionization in QSO Broad Emission-Line Regions
}

\author{
F. Hamann, ${ }^{1}$ J.C. Shields, ${ }^{2}$ R. D. Cohen, ${ }^{1}$ V.T. Junkkarinen, ${ }^{1}$ and \\ E. M. Burbidge ${ }^{1}$ \\ ${ }^{1}$ Center for Astrophysics and Space Sciences, University of California \\ at San Diego, La Jolla, CA 92093, USA \\ ${ }^{2}$ Steward Observatory, University of Arizona, Tucson, AZ 85721, USA
}

\begin{abstract}
We describe two ongoing studies of QSO broad emissionline regions (BELRs). The first employs the $\mathrm{N} \vee \lambda 1240 / \mathrm{He} I \mathrm{I} \lambda 1640$ and $\mathrm{N} v / \mathrm{C}$ IV $\lambda 1549$ line ratios as diagnostics of QSO metallicities. Hamann \& Ferland and Ferland et al. showed that many observed $\mathrm{N} V$ ratios require enhanced $\mathrm{N}$ abundances and $Z>Z_{\odot}$. Here we present new measurements of large line ratios at redshifts $z>4$, which indicate super-solar abundances within $\sim 1$ Gyr of the Big Bang (for $q_{0} \approx 0.5$ ). We also note that the $\mathrm{N} \vee$ line is relatively stronger in more luminous QSOs, in contrast to the well-known Baldwin effect in Ly $\alpha, \mathrm{C}$ IV, and O VI $\lambda 1034$. This unusual behavior in $\mathrm{N} v$ could be due to a luminosity-metallicity correlation among QSOs that is coupled to a mass-metallicity relation in their host galaxies.

Our second study involves Ne VIII $\lambda 774$ as a probe of highly ionized gas. We show that a broad emission feature near $774 \AA$ is common in QSOs. Photoionization models indicate that Ne VIII is the most likely identification for this feature. The models also indicate that the NeVIII emitting gas covers $>40 \%$ of the continuum source, has a total hydrogen column density of $N_{\mathrm{H}}>10^{22} \mathrm{~cm}^{-2}$ (for solar abundances) and an ionization parameter of $U>5$ (for a nominal QSO continuum shape). This gas would be an X-ray "warm" absorber - with $\mathrm{O}$ VII-O VIII bound-free edges - if it lies along our line-of-sight to the $\mathrm{X}$-ray continuum source.
\end{abstract}

\section{N V $\lambda 1240$ and QSO Metal Abundances}

Strong emission lines due to heavy elements in QSOs imply some degree of chemical enrichment out to redshifts of nearly 5. However, extracting quantitative abundance information from the lines is notoriously difficult. Early efforts using ratios of semi-forbidden lines revealed at least occasional nitrogen overabundances (compared to $\mathrm{C}$ and $\mathrm{O}$ ) and total metallicities above solar (Shields 1976; Osmer 1980). These studies were limited, however, by the weakness of the lines and the pitfalls of collisional quenching (also Uomoto 1984). Hamann \& Ferland $(1992,1993)$ and Ferland et al. (1996) recently applied both chemical evolution and photoionization calculations to the analysis of several strong permitted lines. Their evolution models demonstrate the importance of nitrogen 
as a tracer of the enrichment because of the predominance (at least at high $Z$ ) of 'secondary' processing, which causes the $\mathrm{N}$ abundance to increase roughly as the square of the total metallicity (see also Shields 1976). The photoionization calculations show that the $N \vee \lambda 1240 /$ He II $\lambda 1640$ flux ratio can provide robust lower limits on the $\mathrm{N} / \mathrm{He}$ abundance and thus the overall metallicity. $\mathrm{N} v$ forms within the $\mathrm{He}^{++}$recombination zone and, although $\mathrm{NV}$ can be relatively weak in many circumstances, it is not possible to produce strong $\mathrm{N} \mathrm{V}$ without significant He II emission. The same calculations show that N V/C IV $\lambda 1549$ can constrain the $\mathrm{N} / \mathrm{C}$ abundance, although this line ratio is more sensitive to the uncertain geometry and physical conditions because the emission regions are not necessarily co-spatial.

\subsection{Recent Results}

We obtained spectra of $25 z \gtrsim 4$ QSOs with the Multiple Mirror Telescope, spanning wavelengths from shortward of $1200 \AA$ to longward of $1700 \AA$ in the QSO rest frame (see Shields et al., this volume; also Shields \& Hamann 1996). Figure 1 shows preliminary measurements of the $\mathrm{N} v / \mathrm{He}$ II and N v/CIV line flux ratios for 15 of the $25 z \gtrsim 4$ sources. The Figure also shows line ratios from the literature for QSOs at lower redshifts (see Hamann \& Ferland 1993). The data from the literature are incomplete for redshifts $2 \lesssim z \lesssim 4$ in the sense that lower limits were not usually reported for lines not detected. In particular, the He II line is often absent while $\mathrm{N} v$ is detected, and we expect that more large $\mathrm{NV} / \mathrm{He}$ Il ratios would appear at these redshifts in a well-measured sample.

Many of the line ratios in Fig. 1 are considerably larger than expected for solar abundances. The dotted lines in the Figure show the ratios predicted by photoionization models for solar abundances and BELR conditions (i.e., density, ionizing flux, and continuum shape) that nearly maximize these ratios (Hamann \& Ferland 1993). Extensive photoionization calculations (also Ferland et al. 1996) show that much smaller ratios (by $>1 \mathrm{dex}$ ) can occur naturally in the BELR, while 'tuning' the model parameters can yield ratios up to $\sim 2$ times higher than the dotted lines in the Figure. However, it is not possible to explain the upper envelope of measured line ratios (e.g., N v/He II) unless nitrogen is relatively over-abundant and the overall metallicity is above solar. In some cases (Ferland et al. 1996), the derived lower limits are $Z \geq 5 Z_{\odot}$. These results are supported by independent evidence for $Z>Z_{\odot}$ from absorption lines that are intrinsic to QSO environments, such as the broad absorption lines (BALs) and at least some of the so-called associated $\left(z_{a} \approx z_{e}\right)$ systems (see Hamann 1996 and references therein).

Figure 1 shows that the largest $\mathrm{N} v$ emission ratios occur at redshifts $z>$ 1. The underlying relationship might actually be with the QSO continuum luminosities (Hamann \& Ferland 1993), because luminosity and redshift are well correlated in this sample. Several studies that examine a range of luminosities in limited redshift intervals (Laor et al. 1995; Osmer et al. 1994; Véron-Cetty, Véron, \& Tarenghi 1983) show that the $\mathrm{N} v$ line ratios are, in fact, correlated with luminosity. (We are presently measuring spectra of faint QSOs at the Keck Observatory to define the luminosity behavior at $z \approx 3$.) $\mathrm{N} v$ does not participate in the well-known 'Baldwin effect', which describes an empirical trend for lower line equivalent widths in more luminous objects (Baldwin 1977; Kinney et al. 

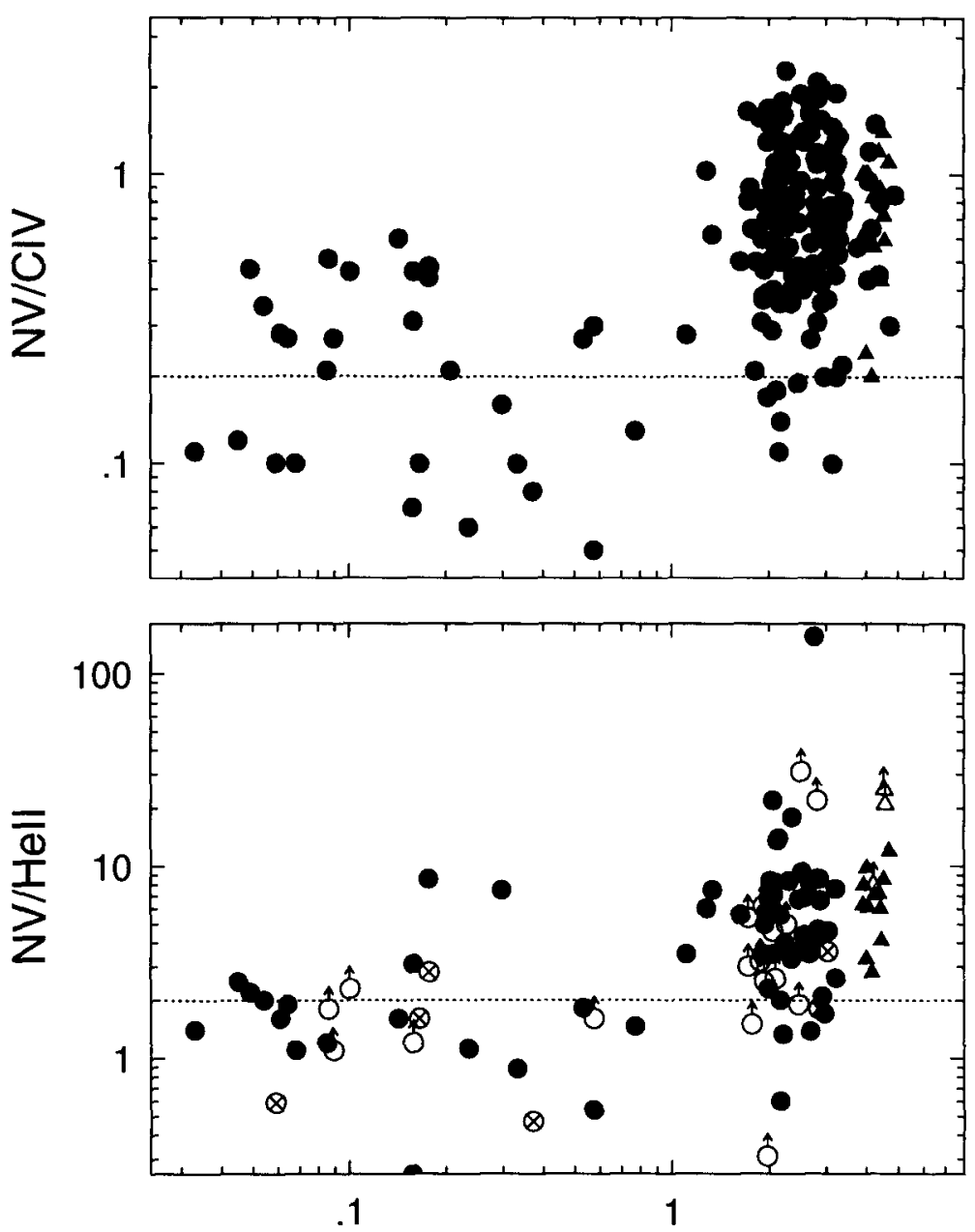

Redshift

Figure 1. Measured line ratios in QSOs. The triangles are the new MMT data for $z>4$. Open symbols indicate lower limits. The dotted lines define the threshold above which super-solar abundances are required by standard photoionization models. 
1990; Zheng et al. 1995). Although there is no consensus explanation for this effect, it is not clear why it should be absent in $\mathrm{N} \mathrm{v}$ but strong in lines such as $C_{I V} \lambda 1549$ and $\mathrm{O}$ VI $\lambda 1034$, which have the same electronic structure as $\mathrm{N} V$ and bracket $\mathrm{N} \mathrm{V}$ in ionization. We suggest that the unusual luminosity behavior of $\mathrm{N} \mathrm{V}$ is due to more enhanced nitrogen abundances and higher overall metallicities in more luminous sources.

\subsection{QSO Metallicities and Host-Galaxy Evolution at High Redshift}

The origin of the metals in QSOs is not known. However, gas-phase metallicities up to $\sim 10 Z_{\odot}$ are consistent with the normal stellar enrichment of a well-mixed interstellar medium in the cores of massive galaxies. The stars in those environments today exhibit a range of metallicities distributed about mean values of typically $\sim 1$ to $\sim 3 Z_{\odot}$. Simple enrichment models indicate that the gas-phase abundances near the end of the star formation episode (when the gas is nearly exhausted) should be several times larger than these stellar means - consistent with the high- $Z$ tail in the stellar distributions (cf. Tinsley 1980; Rich 1990). Although the high- $Z$ gas might not remain in galaxies today - having been ejected in a galactic wind or consumed by the black hole - it could dominate QSO environments at early epochs.

In the local Universe, there is a well-known correlation between galaxy masses (luminosities) and metallicities (cf. Zaritsky, Kennicutt, \& Huchra 1994 and references therein). Metal-rich stellar populations are found only in massive galaxies. This galactic mass-metallicity relation could explain the tentative luminosity-metallicity trend among the QSOs, if QSO luminosities are somehow coupled to the host galaxy's mass. In any case, if the high QSO metallicities are due to normal stellar enrichment, as discussed above, then (at least) the cores of massive galaxies are highly evolved at redshifts $z>4$, within about $1 \mathrm{Gyr}$ of the Big Bang (for $q_{0} \approx 0.5$ ). If the stellar initial mass functions do not excessively favor high-mass stars, the galaxy components that feed metals into QSOs must have already converted most of their mass into stars and stellar remnants before the QSOs 'turn on' (or become observable).

\section{Broad Ne VIII $\lambda 774$ Emission}

The ionization energy required for Ne VIII $\lambda 774(207 \mathrm{eV})$ is nearly twice as large as for $\mathrm{O}$ VI $\lambda 1034(114 \mathrm{eV})$ and more than four times larger than $\mathrm{C}$ IV $(48 \mathrm{eV})$. For BEL clouds photoionized by a standard QSO continuum, significant Ne VIII $\lambda 774$ emission requires ionizing fluxes (i.e., ionization parameters) at least 10 times larger than needed for $\mathrm{OVI}$ and 100 times larger than for CIV (Hamann et al. 1995a). Measurements or upper limits on broad Ne viII $\lambda 774$ are therefore important, not only to probe the highly ionized components of the BELR, but also to study the possible relationship of this component to other high-ionization phenomena, such as X-ray 'warm' absorbers and the source of the near-UV continuum (i.e., the 'big blue bump;' BBB). Warm absorbers (Halpern 1984) are characterized by $\mathrm{O}$ VII-O VIII absorption edges near $0.8 \mathrm{keV}$ or by $\mathrm{X}$-ray continuum opacities that vary inversely with the X-ray flux. Hamann et al. (1995a) showed that the Ne viII-emitting gas would produce a warm absorber if it lay along our line-of-sight to the continuum source. The BBB is generally 
attributed to thermal emission from either an optically thick accretion disk or a more distant optically thin region. Hamann et al. (1995a) showed that the temperatures needed for NeviII emission could be comparable to the BBBemitting gas, although the Nevill region itself is not sufficient to produce the BBB.

Hamann et al. (1995b) studied a sample of 5 QSOs from the Hubble Space Telescope (HST) Absorption Line Snapshot Survey (Absnap) to estimate the strength and character of Ne VIII $\lambda 774$ emission. That sample includes radioloud and radio-quiet QSOs with (1) emission redshifts that include Ne VIII and O VI $\lambda 1034$ between the observed wavelengths of $\sim 1350$ to $\sim 2400 \AA$ (i.e., $z_{e m}$ between $\sim 0.8$ and $\sim 1.4),(2)$ no Lyman-limit absorption across the Ne VIII wavelength, and (3) 'measurable' O VI emission. The last selection criterion serves mainly as a signal-to-noise discriminator, although it could introduce a bias toward sources with more high-ionization gas. The sources were not chosen based on their Ne VIII characteristics. Nonetheless, 3 of the 5 sources, and possibly all five, have a broad emission feature near $774 \AA$. Photoionization calculations by Hamann et al. (1995a) show that Ne VIII is the most likely identification for this feature.

Here we supplement that work with new and archival spectra obtained with $H S T$. We derive an additional sample of 6 QSOs from the archived Absorption Line Key Project spectra, using the same selection criteria as for the Absnap sample above. We reobserve 2 of the 5 Absnap sources to improve the signal-tonoise ratio across Ne viII $\lambda 774$. We also discuss spectroscopy of another bright QSO not included in these samples, PG 1522+101 (previously reported by Cohen et al. 1995).

\subsection{Mean Spectra}

Figure 2 plots mean spectra of the 5 Absnap sources, the 6 Key-Project sources, and the total sample of 11 . The rest wavelengths were derived from the peak centroid of $\mathrm{O}$ VI $\lambda 1034$, assuming that feature is due entirely to $\mathrm{O} V \mathrm{~V}$ at $1033.8 \AA$. The spectra are shown normalized to unity in the continuum by fitting with loworder polynomials. The dotted line in the Figure shows the fit to the continuum and the Ly $\alpha$ emission profile. The fit to Ly $\alpha$ is based on the line's peak and blue-side profile and is symmetric about $1217.5 \AA$. Note that the results for $\mathrm{C}_{\text { III }}{ }^{*}$, Ly $\alpha$ and $N \mathrm{v}$ derive from a subset of each sample because not all of the spectra include these wavelengths.

The Nevill line is clearly present in both the mean and median spectra. (Also note the presence of the excited state line $\mathrm{C}_{1 I}{ }^{*} \lambda 1176$, discussed by Laor et al. in this volume). Table 1 lists the line centroids $\left(\lambda_{\text {rest }}\right)$ and rest equivalent widths (REWs) measured from the total mean spectrum in Fig. 1. The line blends centered on $\mathrm{O} V \mathrm{~V}$ and Ly $\alpha$ were divided among the components by simply integrating the flux above the dotted line in Fig. 1 between the wavelengths indicated by $\Delta \lambda$ in the Table. The broad unidentified feature at $\sim 1070 \AA$ was also noted by Laor et al. (1995).

\subsection{Some Individual Spectra}

Figure 3 shows our attempts to fit two well-measured NevIII $\lambda 774$ profiles in PKS 0355-483 and PG 1522+101, using CIV $\lambda 1549$ as a template. The two 


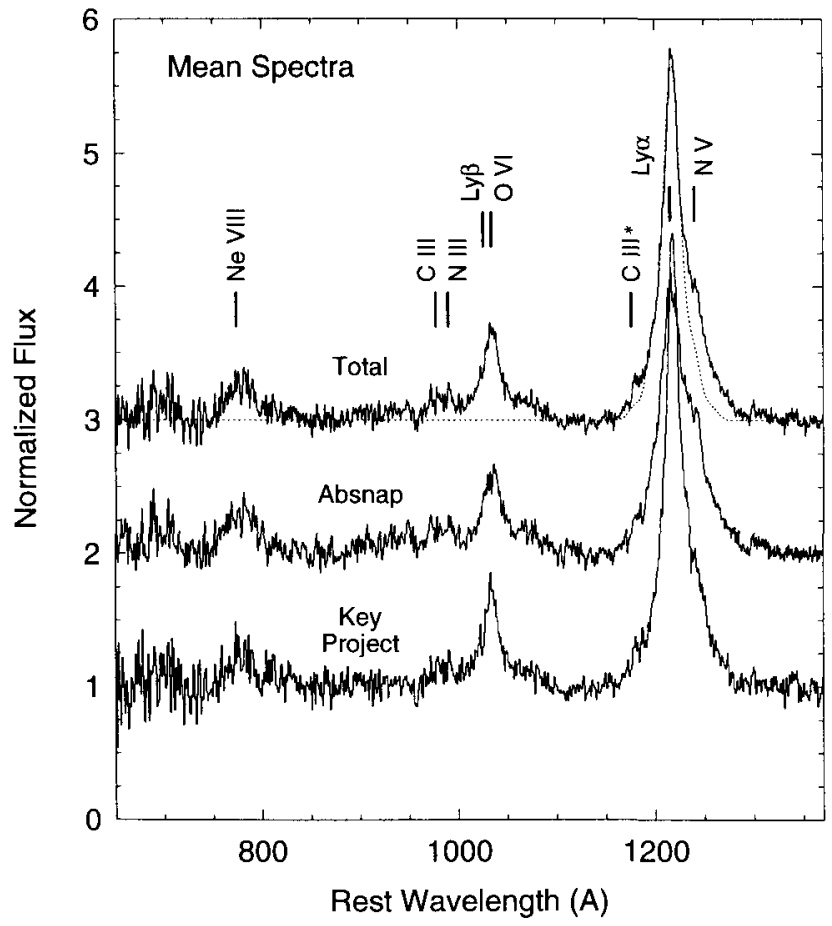

Figure 2. Normalized mean spectra showing Ne VIII $\lambda 774$ and other emission lines. The upper spectra are shifted by adding 1.0 and 2.0 .

Table 1. Mean Line Parameters

\begin{tabular}{lrrc}
\hline \hline \multicolumn{1}{c}{ Line } & $\begin{array}{c}\lambda_{\text {rest }} \\
(\AA)\end{array}$ & $\begin{array}{r}\text { REW } \\
(\AA)\end{array}$ & \multicolumn{1}{c}{$\begin{array}{c}\Delta \lambda \\
(\AA)\end{array}$} \\
\hline Ne VIII $\lambda 774$ & 776.8 & 8.7 & $\ldots$ \\
C III $\lambda 977$ & 976.0 & 2.5 & $968.0-983.5$ \\
N III $\lambda 990$ & 991.4 & 2.1 & $983.5-997.5$ \\
O VI $\lambda 1034$ & 1032.6 & 17.4 & $997.5-1059.5$ \\
$\quad ? ? ?$ & 1070.3 & 3.8 & $1059.5-1084.5$ \\
C III $\lambda 1176$ & 1177.5 & $2.3:$ & $1164.0-1190.0$ \\
Ly $\alpha \lambda 1216$ & 1217.5 & 74.8 & $\ldots$ \\
N V $\lambda 1240$ & 1245.1 & 8.1 & $1227.0-1256.0$ \\
\hline
\end{tabular}



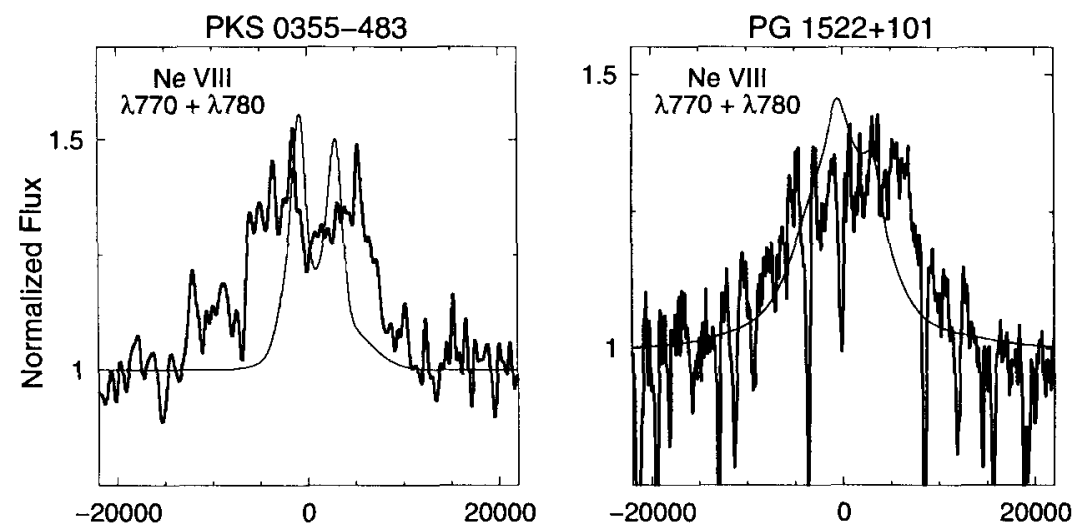

Velocity $(\mathrm{km} / \mathrm{s})$

Figure 3. Fits (thin lines) to the normalized NeviII $\lambda 774$ profiles (bold lines) using CIV $\lambda 1549$ as a template. Zero velocity corresponds to $774.4 \AA$.

components of the Nevill doublet $(770$ and $780 \AA)$ were assigned the same redshift and profile as the CIV line in each spectrum. The only free parameters in the fits were the line strengths. In both cases, the poor fits suggest that the Ne VIII lines are broader than CIV. More data are needed to determine if this is a general property of Ne VIII $\lambda 774$ emission.

\subsection{Ne VIII $\lambda 774$ and the Highly Ionized BELR}

We conclude that broad Ne VIII $\lambda 774$ emission is common in QSOs, with a mean strength of $\sim 50 \%$ of $\mathrm{O}$ VI $\lambda 1034$. The Ne VIII emitting gas identifies a previously unknown, highly ionized component of the BELR. Photoionization calculations (Hamann et al. 1995a) indicate that the ionization parameter (the dimensionless ratio of hydrogen-ionizing photon to hydrogen particle densities $U$ ) must exceed 5 in this region for a nominal QSO continuum shape. The calculations also show that the Ne VIII emitting gas must cover $\gtrsim 40 \%$ of the continuum source and have a total hydrogen column density $N_{\mathrm{H}} \gtrsim 10^{22} \mathrm{~cm}^{-2}$ (for solar metal abundances). This gas would be an X-ray warm absorber if it lay along our line of sight to the continuum source.

Acknowledgments. Thanks to M. Crenshaw, G. J. Ferland, K. T. Korista, H. Netzer, and G. Shields for useful discussions, and L. Zuo and D. Tytler for help with the Absnap data. The line fits were performed using the software package Specfit, developed by G. A. Kriss. This work was supported by NASA grants NAG 5-1630 and NAG 5-3234, and by STScI through the Guest Observer program. 


\section{References}

Baldwin, J. A. 1977, ApJ, 214, 679.

Cohen, R. D., Beaver, E. A., Burbidge, E. M., Diplas, A., Hamann, F., Junkkarinen, V.T., \& Lyons, R. 1995 in The Analysis of Emission Lines, ed. M. Livio, STScI Symposium, (Cambridge: New York).

Ferland, G.J., Baldwin, J.A., Korista, K.T., Hamann, F., Carswell, R.F., Phillips, M., Wilkes, B., \& Williams, R. E. 1996, ApJ, 461, 683.

Halpern, J. P. 1984, ApJ, 281, 90.

Hamann, F. 1996, ApJS, in press.

Hamann, F., \& Ferland, G. J. 1992, ApJL, 391, L53.

Hamann, F., \& Ferland, G. J. 1993, ApJ, 418, 11.

Hamann, F., Shields, J.C., Ferland, G.J., \& Korista, K.T. 1995a, ApJ, 454, 688.

Hamann, F., Zuo, L., \& Tytler, D. 1995b, ApJ, 444, L69.

Kinney, A. L., Rivolo, A. R., \& Koratkar, A.P. 1990, ApJ, 357, 338.

Laor, A., Bahcall, J. N., Jannuzi, B.T., Schneider, D.P., \& Green, R.F. 1995, ApJS, 99, 1.

Osmer, P.S. 1980, ApJ, 237, 666.

Osmer, P.S., Porter, A. C., \& Green, R.F. 1994, ApJ, 436, 678.

Rich, R. M. 1990, ApJ, 362, 604.

Shields, G. A., 1976, ApJ, 204, 330.

Shields, J. C., \& Hamann, F. 1996, Rev. Mex. Astr. Astrof., (Conference Series), in press.

Tinsley, B. M. 1980, Fund. Cos. Phys., 5, 287.

Uomoto, A. 1984, ApJ, 284, 497.

Véron-Cetty, M.-P., Véron, P., \& Tarenghi, M. 1983, A\&A, 119, 69.

Zaritsky, D., Kennicutt, R. C., Jr., \& Huchra, J.P. 1994, ApJ, 420, 87.

Zheng, W., Kriss, G. A., \& Davidson, A.F. 1995, ApJ, 440, 606. 